\title{
Isolation of Coxiella burnetii in patients with nonspecific febrile illness in South Korea
}

\author{
Seung Hun Lee ${ }^{1,2}$, Jae Hoon Lee ${ }^{3}$, Sungdo Park', Hae Kyung Lee ${ }^{1}$, Seon Do Hwang ${ }^{1}$, Hye Won Jeong ${ }^{4}$, \\ Jung Yeon $\mathrm{Heo}^{5^{*+}}$ and Yeong Seon Lee ${ }^{1^{*+}}$
}

\begin{abstract}
Background: The number of human Q fever cases in South Korea has been rapidly increasing since 2015. We report the first isolation of Coxiella burnetii in Korea in two patients who initially presented with non-specific febrile illness and were finally diagnosed with acute Q fever in South Korea.

Case presentation: Two adult patients with fever had serologic tests against C. burnetii initially negative, and polymerase chain reaction against $16 \mathrm{~S}$ rRNA using whole blood was also negative. After bacterial amplification of $C$. burnetii in immune-depressed mice, we isolated C. burnetii from patients with acute Q fever. The isolates KZQ2 and KZQ3 were confirmed by polymerase chain reaction, nucleotide sequence analysis, and morphologic observation using a transmission electron microscope.
\end{abstract}

Conclusions: These results can help us understand the clinical and epidemiologic features of $Q$ fever in South Korea.

Keywords: Coxiella burnetii, Q fever, Culture isolation, molecular sequencing, electron microscopy

\section{Background}

Acute $\mathrm{Q}$ fever is an infectious zoonotic disease that is characterized by a sudden onset of fever, headache, and atypical pneumonia. It is primarily diagnosed via a serologic test, and paired samples from the acute and convalescent phases taken several weeks apart are required to confirm the diagnosis. Because of these nonspecific symptoms and diagnostic challenges, acute $\mathrm{Q}$ fever has been considered an under-recognized and underdiagnosed infectious disease, particularly in non-endemic or non-epidemic areas. Although acute $\mathrm{Q}$ fever is generally self-limiting and resolves in < 14 days, even in untreated patients, it can lead to public health concerns, such as

\footnotetext{
*Correspondence: jyeon78@naver.com; yslee0707@gmail.com

†Jung Yeon Heo and Yeong Seon Lee contributed equally to this work.

${ }^{5}$ Division of Infectious Diseases, Ajou University School of Medicine,

Suwon-si, Kyeonggi-do 16499, South Korea

'Division of Bacterial Disease Research, Center for Infectious Disease

Research, Korea National Institute of Health, Osongsaengmyeong 2-ro, Cheongju-si 28159, South Korea

Full list of author information is available at the end of the article
}

the large-scale outbreaks in the Netherlands, and has the potential of being used as a bioterrorism agent $[1,2]$. Furthermore, chronic Q fever can develop after symptomatic or asymptomatic acute infection; for example, as endocarditis or vascular infection. Chronic $Q$ fever develops in approximately $<5 \%$ of persons with acute infection [3]. It is therefore necessary to understand the microbiologic and molecular characteristics of Coxiella burnetii in patients with acute $\mathrm{Q}$ fever from different geographic areas. However, it is usually not possible to isolate $C$. burnetii from acutely infected patients because of the difficulty in obtaining suitable samples or handling them safely. Nevertheless, to control diseases and understand epidemiologic features in during a $\mathrm{Q}$ fever epidemic, we should understand the microbiologic characteristics of circulating C. burnetii through isolation.

A rapid increase in the annual incidence of $\mathrm{Q}$ fever has recently occurred in South Korea, with 0.16 cases per 100,000 persons in 2016 and 0.19 cases per 100,000 persons in 2017. Compared to 0.02 cases per 100,000 
persons in 2008, this represents a greater than eight-fold increase. The Korea Centers for Disease Control and Prevention reported that the number of confirmed cases of Q fever was 8 in 2014, 27 in 2015, 81 in 2016, 96 in 2017, and 163 in 2018. Although the number of confirmed $\mathrm{Q}$ fever cases has been increasing, there have been no cases on the isolation of $C$. burnetii from human blood in Korea [4]. Here, we report the isolation of C. burnetii in two patients who initially presented with non-specific febrile illness but were finally diagnosed with acute $\mathrm{Q}$ fever.

\section{Case presentation}

Patient 1, a 32-year-old man office worker living in the outskirts of Cheongju-si, Chungcheongbuk-do, South Korea, was admitted to a hospital with a 5-day history of fever and headache in March 2016. On physical examination, he had no remarkable findings except for a body temperature of $39.6{ }^{\circ} \mathrm{C}$. Laboratory tests showed normal platelet $\left(217 \times 10^{3} / \mu \mathrm{L}\right)$ and white blood cell $(5720 / \mu \mathrm{L})$ counts with elevated C-reactive protein $(8.27 \mathrm{mg} / \mathrm{dL})$, aspartate aminotransferase $(71 \mathrm{IU} / \mathrm{L})$, and alanine transaminase (76 IU/L) levels. Although intravenous ceftriaxone was initiated as an empiric antibiotic treatment for the febrile illness, no bacterial or fungal organisms were isolated in the blood samples. Because of the persistent fever ( $>7$ days) despite antibiotic treatment, a serum sample was collected for specific $C$. burnetii antibody and nucleic acid detection on hospital day 4 . The patient had no history of animal contact. Although ceftriaxone was only administered for 5 days, the patient was discharged in an afebrile state after 9 days in the hospital.

Patient 2, a previously healthy 65-year-old man, visited an outpatient clinic in May 2016 with a 1-month history of fever and general weakness. He lived in Buan-gun, Cheollabuk-do, South Korea and worked as a dairy cattle raiser. Physical examination revealed an elevated body temperature of $38.5^{\circ} \mathrm{C}$ but no other remarkable findings. A complete blood count showed mild thrombocytopenia (platelet count, $142 \times 10^{3} / \mu \mathrm{L}$ ) and a normal white blood cell count $(4050 / \mu \mathrm{L})$ with $59.8 \%$ neutrophils. Blood biochemistry revealed elevated C-reactive protein $(3.11 \mathrm{mg} / \mathrm{dL})$, aspartate aminotransferase $(44 \mathrm{IU} / \mathrm{L})$, and alanine transaminase $(40$ IU/L) levels. Given his history of animal contact, a blood sample for C. burnetii antibody testing and isolation was collected before administering oral doxycycline as an empirical antibiotic treatment. After doxycycline was prescribed for 7 days, his symptoms gradually improved.

We used an indirect fluorescent antibody (IFA) assay from a commercial kit (IF0200G, IF0200M, Focus Diagnostics, Cyprus, California, USA). The initial serum samples, which were obtained on about days 9 and 30 of symptom onset, were negative for phase II IgG and IgM against C. burnetii. Furthermore, the phase I IgG and IgM antibodies were negative. To detect the gene of the causative antigen, we performed nested polymerase chain reaction (PCR) assays on blood samples of the patients and primers specific to the 16S rRNA of Anaplasma phagocytophilum, Ehrlichia chaffeensis, C. burnetii, and the outer membrane protein (omp) of Rickettsia spp. All PCR test results were negative; however, after 9 and 14 weeks, for patients 1 and 2 the phase II IgG and IgM titers were $\geq 1: 2048$ and 1:16, respectively, from serum samples in the convalescence phase. The phase I IgG and IgM titers were 1:512 and 1:64 for patient 1 and 1:512 and 1:128 for patient 2 .

To amplify the bacteria, we inoculated $400 \mu \mathrm{L}$ buffy coats from the patients' blood into immune-depressed $\mathrm{BALB} / \mathrm{c}$ mice via the intraperitoneal route. On day 49 post-infection (dpi 49), the spleens of the infected animals that had developed splenomegaly were harvested (Additional file 1: Figure S1). The spleens were homogenized for cell culture and gene identification, and DNA was extracted for PCR analyses of the specific genes described above. The PCR results were positive for $16 \mathrm{~S}$ rRNA, IS1111, and omp-specific genes of C. burnetii.

The nucleotide sequences of the isolates KZQ2 (patient 1) and KZQ3 (patient 2) [16S rRNA (KX825917, KY498541), IS1111 (MG793228, MG793229), and omp (MG836249, MG836250)] were matched to C. burnetii. Comparison of the 16S rRNA nucleotide sequences showed high similarity (99.6-100\%) with C. burnetii sequences (USA: CP001019, USA: D89791, Japan: D89795) (Fig. 1a). The sequences were identical to South Korea: KX825917, KY498541, and AY342037 as well as USA: M21291. The nucleotide sequences of IS1111 were identical to those of C. burnetii (Fig. 1b); those of omp showed $80.30-99.75 \%$ sequence similarity to tick and human sequences from South Korean isolates (tick: AY342038, human: KM11542, MG836249, and MG836250).

Homogenized spleens were infected in the African green monkey kidney epithelial cell line Vero (KCLB-10081) in RPMI1640 medium (GIBCO, Waltham) supplemented with $2 \%$ fetal bovine serum (GIBCO, Waltham) and 0.4 mM L-glutamine (GIBCO, Waltham) and cultured in an incubator at $37^{\circ} \mathrm{C}$ with $5 \% \mathrm{CO}_{2}$; the medium was changed regularly. Before cell passage, the infected cells were examined under a transmission electron microscope after Diff-Quik (Sysmex, Japan) staining and in-house IFA testing.

Bacterial isolation from the animals was performed under Animal Biosafety Level-3 conditions, and cell infections were performed under Biosafety Level 3 


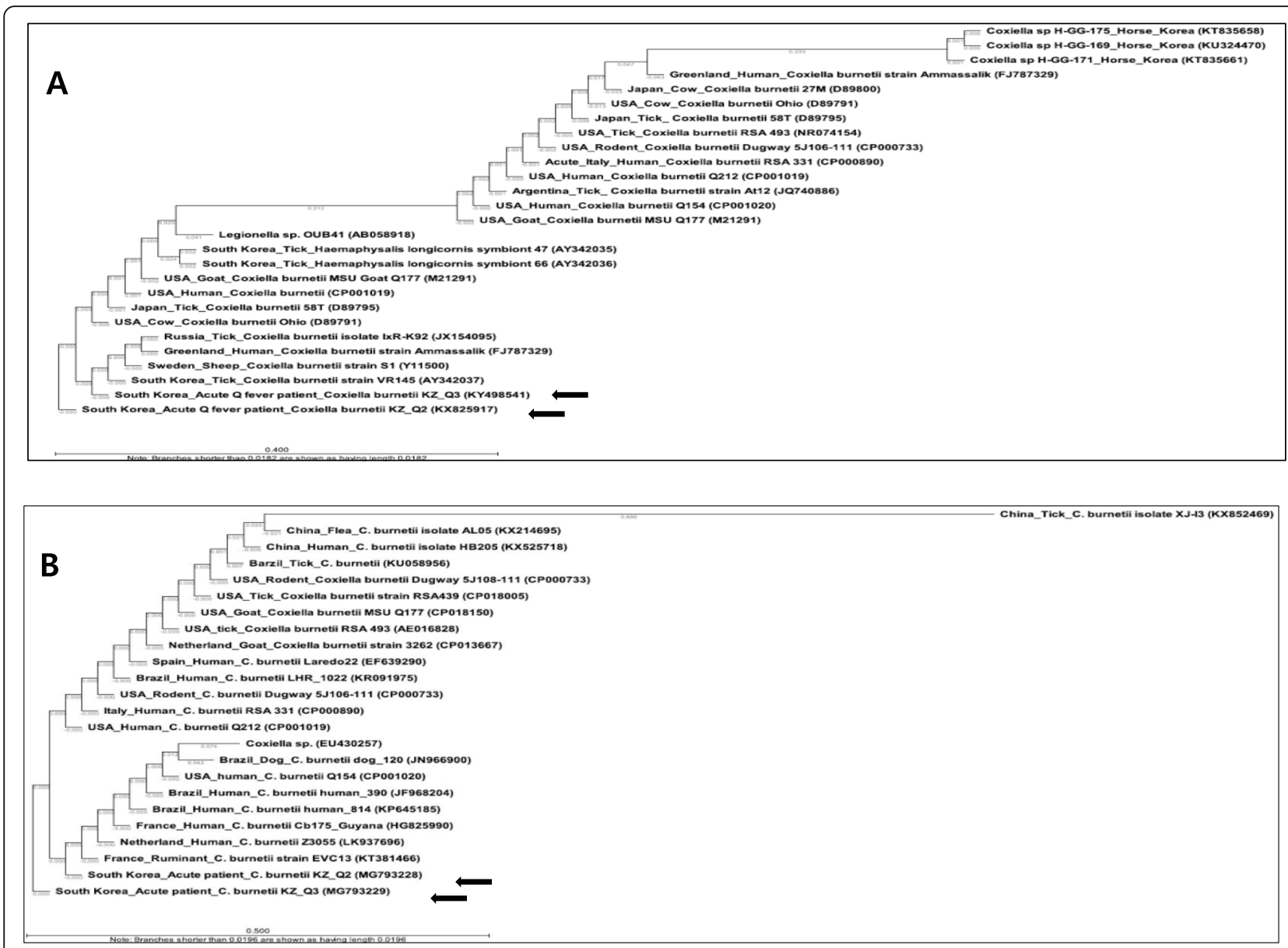

Fig. 1 Partial sequences of the $16 \mathrm{~S}$ rRNA and IS1111 of Coxiella burnetii isolates obtained from two patients with Q fever in South Korea (arrows). a $16 \mathrm{~S}$ rRNA and $\mathbf{b}$ 1S1111. The phylogenetic trees were constructed using the Jukes-Cantor/Neighbor joining methods. The locations, hosts, and GenBank accession numbers are indicated. The branch lengths of the trees show the evolutionary distances, and the numbers on the branches indicate bootstrap support (1000 replicates)

conditions. All protocols using live animals were reviewed and approved by an Institutional Animal Care and Use Committee (IACUC, KCDC-045-16-2A) and we used for four-week-old Balb/c mice were purchased from Orientbio (Seoul, South Korea) which used $\mathrm{CO} 2$ euthanasia.

During cell infection, C. burnetii antigens were confirmed with the in-house IFA method using seropositive serum (Focus Diagnostics, CA) as the primary antibody and human IgG-fluorescein-conjugated isothiocyanate (Focus Diagnostics, CA) as the secondary antibody. Every 2 weeks, the infected cells were passaged. On dpi 3, we observed fluorescent C. burnetii antigens in the cell pellet using the in-house IFA test (Additional file 2: Figure S2). On dpi 10, an evaluation of the morphology of C. burnetii with Diff-Quik (Sysmex, Japan) staining (Additional file 3: Figure S3) and transmission electron microscopy revealed replication of $C$. burnetii in the vacuole within the cytoplasm of the infected cells (Fig. 2).

\section{Discussion and conclusions}

We first isolated C. burnetii from the whole blood of two patients with acute $\mathrm{Q}$ fever who presented with non-specific febrile illness in South Korea. Although the IFA against C. burnetii and PCR of 16S rRNA were initially negative, we were able to isolate $C$. burnetii after bacterial amplification in immunedepressed mice. The C. burnetii isolates KZQ2 and KZQ3 were verified by PCR, sequence analysis, and morphologic observations.

The molecular characteristics of C. burnetii have been previously reported from milk samples, and C. burnetii was detected in ticks, livestock, and humans in South Korea [5-9]. We assume that C. burnetii may be more widespread in South Korea than previously reported. To confirm this suspicion, additional investigations in patients, animals, ticks, and environmental samples are needed.

It is difficult to identify patients with acute $\mathrm{Q}$ fever if they initially have negative serologies against $C$. 

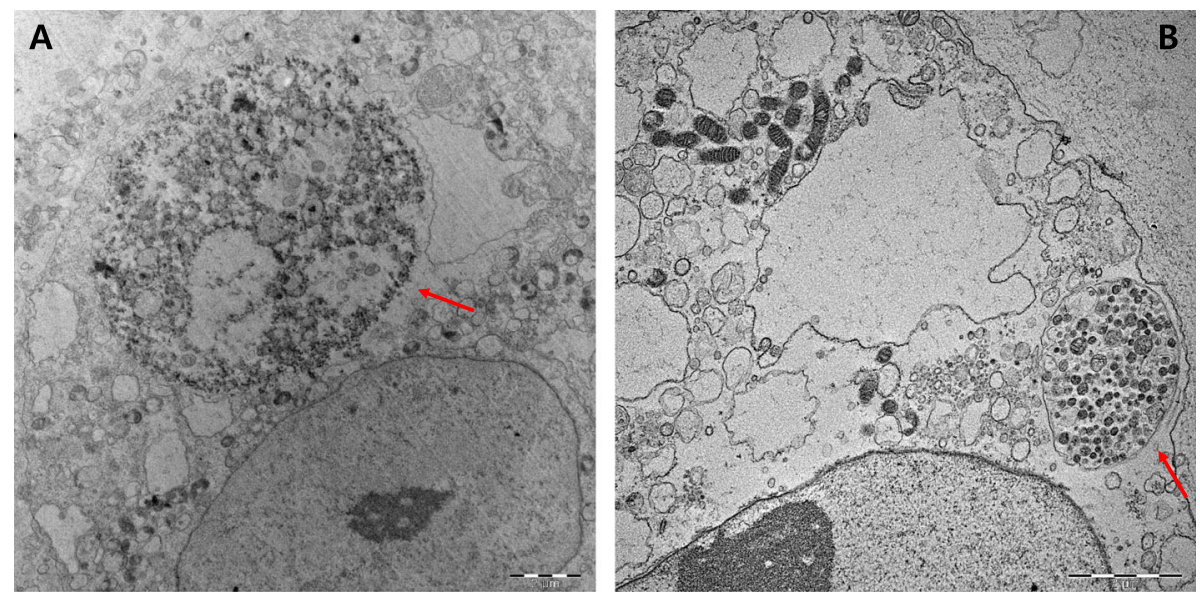

Fig. 2 Morphology of the isolated Coxiella burnetii in Vero cells visualized by transmission electron microscopy. Vero cell monolayers were incubated with homogenates of $C$. burnetii-infected mice spleen, and fresh medium was added. The infected cells were fixated and processed for transmission electron microscopy on post-infection day 60. Bar, $2 \mu \mathrm{m}$. The arrow shows the replicated C. burnetii (a: KZQ2, b: KZQ3)

burnetii. To accurately confirm acute Q fever, serologic tests should be conducted using paired sera from the acute and convalescent stages taken 4-6 weeks apart. However, symptomatic acute $Q$ fever usually presents the duration of fever within 2 weeks $[10,11]$. Without epidemiologic factors such as livestock raiser and slaughterhouse worker, patients with acute $\mathrm{Q}$ fever who have negative serology at the acute stage of illness are more likely to be neglected. This is the reason two cases in our study were confirmed to have acute $\mathrm{Q}$ fever at 9 and 14 weeks after the initial serologic test. Delayed diagnosis also hinders proper monitoring of patients with chronic Q fever. Most physicians usually consider that bacterial infection does not progress to chronic infections. Therefore, no follow up was organized for these patients, and we could not perform echocardiography and monitor their follow-up serology.

Although quantitative PCR could not be performed, we demonstrated bacterial isolation using methods such as TEM, Diff-Quik, IFA, and genetic analysis from cell culture. It may be meaningful that the study is useful because the extant clinical isolate has been obtained from Korean $Q$ fever patient, which will provide a basis for characterizing a human isolate and identifying isolate methods in South Korea.

The human isolates KZQ2 and KZQ3 identified in the present case study are the first isolates from patients with Q fever in South Korea. These results provide a clue to understanding the bacterial characterization of $C$. burnetii isolated from Korean patients and might help healthcare providers understand the clinical and epidemiologic features of these patients. Future studies should analyze the pathogenicity and infectivity of Korean C. burnetii isolates.

\section{Supplementary information}

Supplementary information accompanies this paper at https://doi.org/10. 1186/s12879-020-05130-3.

Additional file 1: Figure S1. The spleen of a Balb/c mouse infected with the patient's buffy coat; day 49; splenomegaly. The spleen is twice as large as the normal.

Additional file 2: Figure S2. Light micrograph of Coxiella burnetii cultured in African green monkey kidney epithelial cell line (Vero) (KZQ2 and KZQ3; Diff-Quik staining). Original magnification ( $A$ and $B ; \times 1000$ ).

Additional file 3: Figure S3. In-house immunofluorescence staining of isolate Coxiella burnetii from patient in African green monkey kidney epithelial cell line (Vero) (dpi 3). Culture preparations stained by IFA using an anti-C. burnetii serum. Green indicates intracytoplasmic inclusions filled with numerous bacteria. Fluorescence magnification $(A-B ; \times 400)$.

\section{Abbreviations}

C. burnetii: Coxiella burnetii; IFA: Indirect fluorescent antibody;

PCR: Polymerase chain reaction; omp: outer membrane protein

\section{Acknowledgments}

None.

\section{Authors' contributions}

Conception and design: YSL, SDH. Acquisition of data: SHL, JYH, JHL. Analysis and interpretation of data: SHL, HKL, YSL, HWJ, SP. Manuscript preparation:

$\mathrm{SHL}, J \mathrm{YH}$. Manuscript approval: all authors.

\section{Funding}

This study was supported by research grants (2017-NI52002-00 and 4838303-210-13) from the Korea Centers for Disease Control and Prevention.

\section{Availability of data and materials}

The data used and/or analyzed during the current study are available from the corresponding author on reasonable request.

\section{Ethics approval and consent to participate}

This study was approved by the Institutional Animal Care and Use Committee (IACUC, KCDC-045-16-2A). Two participants gave their written consent to participate.

\section{Consent for publication}

Written informed consent was obtained from two participants for their data to be used for research and publication. 


\section{Competing interests}

The authors declare that they have no competing interests.

\section{Author details}

'Division of Bacterial Disease Research, Center for Infectious Disease Research, Korea National Institute of Health, Osongsaengmyeong 2-ro, Cheongju-si 28159, South Korea. ${ }^{2}$ Yeosu National Quarantine station, Yeosu-si, Jeollanam-do 58729, South Korea. 'Wonkwang University College of Medicine, Iksan-si, Jeollabuk-do 54538, South Korea. ${ }^{4}$ Chungbuk National University College of Medicine, Cheongju-si, Chungcheongbuk-do 28644, South Korea. ${ }^{5}$ Division of Infectious Diseases, Ajou University School of Medicine, Suwon-si, Kyeonggi-do 16499, South Korea.

Received: 25 July 2019 Accepted: 1 June 2020

Published online: 17 June 2020

\section{References}

1. Schneeberger PM, Wintenberger C, van der Hoek W, Stahl JP. Q fever in the Netherlands - 2007-2010: what we learned from the largest outbreak ever. Med Mal Infect. 2014:44:339-53.

2. Madariaga MG, Rezai K, Trenholme GM, Weinstein RA. Q fever: a biological weapon in your backyard. Lancet Infect Dis. 2003;3:709-21.

3. Fenollar F, Fournier PE, Carrieri MP, Habib G, Messana T, Raoult D. Risks factors and prevention of Q fever endocarditis. Clin Infect Dis. 2001:33:312-6.

4. Q fever patients: Infectious disease statics system assessment [http://www. cdc.go.kr/npt/]. Accessed 25 July 2019.

5. Lee JH, Park HS, Jang WJ, Koh SE, Park TK, Kang SS, et al. Identification of the Coxiella sp. detected from Haemaphysalis longicornis ticks in Korea. Microbiol Immunol. 2004;48:125-30.

6. Kwak W, Chu H, Hwang S, Park JH, Hwang KJ, Gwack J, et al. Epidemiological characteristics of serologically confirmed Q fever cases in South Korea, 2006-2011. Osong Public Health Res Perspect. 2013;4:34-8.

7. Chu H, Yoo SJ, Hwang KJ, Lim HS, Lee K, Park MY. Seroreactivity to Q fever among slaughterhouse workers in South Korea. J Prev Med Public Health. 2017;50:195-200.

8. Seo MG, Ouh IO, Lee SH, Kwak D. Detection and genotyping of Coxiella burnetii in pigs, South Korea, 2014-2015. Emerg Infect Dis. 2016;22:2192-5.

9. Seo MG, Ouh IO, Lee SH, Kim JW, Rhee MH, Kwon OD, et al. Prevalence of Coxiella burnetii in cattle at south Korean national breeding stock farms. PLoS One. 2017;12:e0177478.

10. Anderson A, Bijlmer H, Fournier P-E, Graves S, Hartzell J, Kersh GJ, et al. Diagnosis and management of $Q$ fever-United States, 2013: recommendations from $C D C$ and the $\mathrm{Q}$ fever working group. Morb Mortal Wkly Rep Recomm Rep. 2013;62(3):1-30.

11. Heo JY, Choi YW, Kim EJ, Lee SH, Lim SK, Hwang SD, et al. Clinical characteristics of acute $\mathrm{Q}$ fever patients in South Korea and time from symptom onset to serologic diagnosis. BMC Infect Dis. 2019;19(1):903. https://doi.org/10.1186/s12879-019-4479-0.

\section{Publisher's Note}

Springer Nature remains neutral with regard to jurisdictional claims in published maps and institutional affiliations.

Ready to submit your research? Choose BMC and benefit from:
- fast, convenient online submission
- thorough peer review by experienced researchers in your field
- rapid publication on acceptance
- support for research data, including large and complex data types
- gold Open Access which fosters wider collaboration and increased citations
- maximum visibility for your research: over 100M website views per year
At BMC, research is always in progress.
Learn more biomedcentral.com/submissions

